\title{
Application of Neural Networks for Estimation of Concrete Strength
}

\author{
Jong-In Kim, M.ASCE ${ }^{1}$; Doo Kie Kim, M.ASCE${ }^{2}$; Maria Q. Feng, M.ASCE ${ }^{3}$; and Frank Yazdani, M.ASCE ${ }^{4}$
}

\begin{abstract}
The uniaxial compressive strength of concrete is the most widely used criterion in producing concrete. Although testing of the uniaxial compressive strength of concrete specimens is done routinely, it is performed on the 28th day after concrete placement. At this point, it is too late to make improvements if the test result does not satisfy the required strength. Therefore, the strength estimation before the placement of concrete is highly desirable. This study presents the first effort in applying neural network-based system identification techniques to predict the compressive strength of concrete based on concrete mix proportions. Back-propagation neural networks were developed, trained, and tested using actual data sets of concrete mix proportions provided by two ready-mixed concrete companies. The compressive strengths estimated by the neural networks were verified by laboratory testing results. This study demonstrated that the neural network techniques are effective in estimating the compressive strength of concrete based on the mix proportions. Application of these techniques will contribute significantly to the concrete quality assurance.
\end{abstract}

DOI: $10.1061 /(A S C E) 0899-1561(2004) 16: 3(257)$

CE Database subject headings: Neural networks; Compressive strength; Estimation; Concrete; Mixing.

\section{Introduction}

Concrete is the most widely used structural material for construction today. Traditionally, concrete has been fabricated from a few well-defined components: Cement, water, fine aggregate, coarse aggregate, etc. In concrete mix design and quality control, the strength of concrete is regarded as the most important property. Many other properties of concrete, such as elastic modulus, water tightness or impermeability, resistance to weathering agents, etc. are directly related to the strength. The compressive strength of concrete is many times greater than other types of strength, and a majority of concrete elements are designed to take advantage of the higher compressive strength of the material. Most often, an ultimate target in the mixture design is the 28th day compressive strength. The 28th day compressive strength is usually determined based on a standard uniaxial compression test and is accepted universally as a general index of concrete strength.

The compressive strength of concrete is related to mix proportions and mix preparation techniques, but the result of the compression test of a specimen can be influenced by the shape, dimension, and the boundary conditions of the specimen. Traditionally, concrete mix is designed based on previous experi-

${ }^{1}$ Professor, Dept. of Civil Engineering, Taegu Univ., Kyongsan, Kyongbuk, 712-714, Korea. E-mail: jikim@biho.taegu.ac.kr

${ }^{2}$ Assistant Professor, Dept. of Civil Engineering, Kansan Univ., Kunsan, Jeonbuk, 573-701, Korea. E-mail: kim2kie@ kunsan.ac.kr

${ }^{3}$ Professor, Dept. of Civil and Environmental Engineering, Univ. of California, Irvine, CA 92697-2175. E-mail: mfeng@uci.edu

${ }^{4}$ Professor, Dept. of Civil Engineering and Construction, North Dakota State Univ., Fargo, ND 58105.

Note. Associate Editor: Roberto Lopez-Anido. Discussion open until November 1, 2004. Separate discussions must be submitted for individual papers. To extend the closing date by one month, a written request must be filed with the ASCE Managing Editor. The manuscript for this paper was submitted for review and possible publication on September 24, 2002; approved on June 30, 2003. This paper is part of the Journal of Materials in Civil Engineering, Vol. 16, No. 3, June 1, 2004. (CASCE, ISSN 0899-1561/2004/3-257-264/\$18.00. ences. However, knowledge from previous experiences is limited since all possible combinations of components, proportions, and mixing techniques have not been investigated or published.

Generally, concrete testing procedures are time consuming and experimental errors are inevitable. A typical compression test is performed about 28 days after placing the concrete. Should the test results fall short of the required strength, costly remediation efforts must be undertaken. Therefore, it is important to be able to estimate the compressive strength of concrete before placing it at construction sites. Artificial neural network techniques (hereafter, neural networks) open new possibilities in the classification and generalization of available experimental results to estimate concrete strength from the mix components.

This study presents, for the first time, the application of the neural networks for estimating the compressive strength of concrete. Training and testing patterns of a neural network were prepared using the data sets containing the mix proportions of two ready-mixed concrete companies. The estimated strengths were compared with those tested in the laboratory. It is shown that the neural network can effectively estimate the strength in spite of the complexity and incompleteness of the available data, and it can be used as a new tool by a concrete mix designer to support the decision process and improve its efficiency.

\section{Neural Network-Based Estimation}

\section{Overview of Neural Networks}

The human brain represents the most sophisticated biological neural networks, which are often much more efficient, adaptable, and tolerant than conventional computers in the field of recognition, control, and learning. Although the processing speed of biological neurons is much slower than that of digital computers, the massive parallel processing power of these neurons overcomes their speed deficiency. The development of artificial neural networks was inspired by neuroscience which studies brain, biological neurons, and synapses. Neural networks are intended to mimic 
the behavior of biological learning and the decision-making process without being biologically realistic, in detail. Neural networks represent simplified methods of a human brain and may be used to solve problems that conventional methods with traditional computations find difficult to solve. The first wave of interest emerged after the introduction of simplified neurons, but only in the past few years have neural networks emerged as a new practical alternative to mainly deal with a pattern recognition in many fields, such as biological, business, environmental, financial, manufacturing, medical, and military. In civil engineering, neural networks have been applied to the detection of structural damage (e.g., Feng and Bahng 1999), structural system identification (e.g., Chen and Shah 1992; Feng and Kim 1998), the modeling of material behavior, structural optimization (e.g., Adeli and Park 1995), structural control (e.g., Chen et al. 1995), ground water monitoring, and concrete mix proportions (e.g., Oh et al. 1999).

Neural networks are networks of many simple processes, which are called units, nodes, or neurons, with dense parallel interconnections. The connections between the neurons are called synapses. Each neuron receives weighted inputs from other neurons and communicates its outputs to other neurons by using an activation function. Thus, information is represented by massive cross-weighted interconnections. Neural networks might be single-or multilayered. The single-layer neural networks present processing units of the neural networks, which take input from the outside of the networks and transmit their output to the outside of the networks; otherwise, the neural networks are considered multilayered. The basic methodology of neural networks consists of three processes: Network training, testing, and implementation. The connection weights of the neural network are adjusted through the training process, while the training effect is referred to as learning. Training of neural networks usually involves modifying connection weights by means of a learning rule. The learning process is done by giving weights and biases computed from a set of training data or by adjusting the weights according to a certain condition. In other words, neural networks learn from examples and exhibit some capability for generalization beyond the training data. Then, other testing data are used to check the generalization. The initial weights and biases joining nodes of an input layer, hidden layers, and an output layer are commonly assigned randomly. The weights and biases are changed for the output of networks to match required data values. As input data are passed through hidden layers, sigmoidal activation functions are generally used. During the training procedure, the data are selected uniformly. A specific pass is completed when all data sets have been processed. Generally, several passes are required to

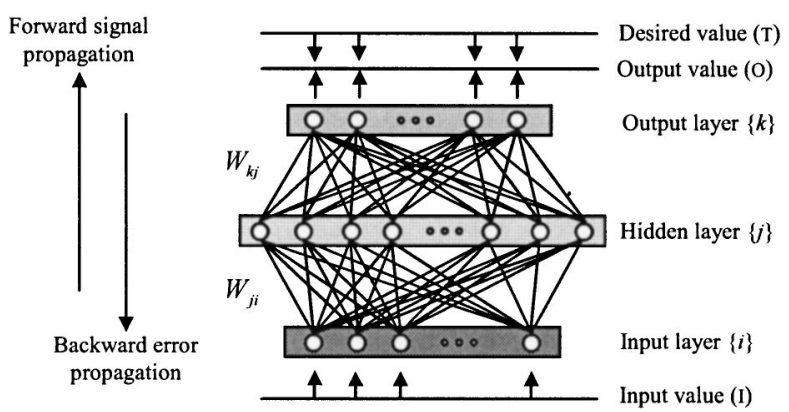

Fig. 1. Architectural layout of back-propagation neural networks

attain a desired level of estimation accuracy. The final sets of weights and biases comprise the long-term memory, or synapses, of respective events. Consequently, learning corresponds to determining the weights and biases associated with the connections in the networks. The back-propagation networks were used in this study. Fig. 1 presents a simple architectural layout of the backpropagation networks that consist of an input layer, a hidden layer, an output layer, and connections between them.

The corresponding architecture for the back-propagation learning incorporates both the forward and the backward phases of the computations involved in the learning process. The learning mechanism of the back-propagation networks is a generalized delta rule that performs a gradient descent on the error space to minimize the total error between the actual calculated values and the desired ones of an output layer during modification of connection weights. In other words, a least mean square procedure is carried out to find the values of the connection weights that minimize the error function by using a gradient descent method. The training is accomplished in an iterative manner. The training procedure is summarized by the following steps:

- Step 1: Assign initial values to connection weights $W_{j i}$ and $W_{k j}$, and to biases $\theta_{j}$ and $\theta_{k}$.

- Step 2: Input values net ${ }_{p i}$ become activations on the input neurons in an input layer.

- Step 3: Training and testing patterns are prepared.

- Step 4: Calculate input values of a hidden layer $j$, net $_{p j}$, using the output values of an input layer $i, O_{p i}$, connection weights $W_{j i}$, and biases $\theta_{j}$ between an input layer $i$ and a hidden layer $j$. Then, the output values of a hidden layer $j, O_{p j}$, are derived from net ${ }_{p j}$ and activation function $f(\cdot)$ :

Table 1. Material Properties of Concrete

\begin{tabular}{|c|c|c|c|}
\hline \multicolumn{2}{|c|}{ Properties of material } & \multicolumn{2}{|c|}{ Experimental data } \\
\hline Property & Content & Company A & Company B \\
\hline \multirow[t]{4}{*}{ Specific gravity } & Cement & 3.14 & 3.15 \\
\hline & Natural sand $\left(\mathrm{s}_{1}\right)$ & 2.59 & 2.58 \\
\hline & Crushed sand $\left(\mathrm{s}_{2}\right)$ & 2.51 & - \\
\hline & Coarse aggregate & 2.64 & 2.63 \\
\hline \multirow[t]{3}{*}{ Fineness modulus } & Natural sand $\left(s_{1}\right)$ & 3.30 & 2.70 \\
\hline & Crushed sand $\left(\mathrm{s}_{2}\right)$ & 2.25 & - \\
\hline & Coarse aggregate & 6.53 & 6.60 \\
\hline
\end{tabular}

Admixtures

Air-entraining admixtures

AE water reducing (Standard)

AE water reducing (Standard)

Note: $\mathrm{AE}=$ Air entraining. 
Table 2. Samples of Specified Concrete Mix Proportions of Company A for Training

\begin{tabular}{|c|c|c|c|c|c|c|c|c|c|}
\hline \multirow{2}{*}{$\begin{array}{l}\text { Specified } \\
\text { strength } \\
(\mathrm{MPa})\end{array}$} & \multirow{2}{*}{$\begin{array}{l}\text { Slump } \\
(\mathrm{cm})\end{array}$} & \multirow{2}{*}{$\begin{array}{l}\text { Water-cement } \\
\text { ratio }\end{array}$} & \multirow{2}{*}{$\begin{array}{c}\text { Fine } \\
\text { aggregate } \\
\text { percentage }(\%)\end{array}$} & \multirow{2}{*}{$\begin{array}{c}\text { Unit } \\
\text { water content } \\
\left(\mathrm{kN} / \mathrm{m}^{3}\right)\end{array}$} & \multirow{2}{*}{$\begin{array}{c}\text { Unit } \\
\text { cement content } \\
\left(\mathrm{kN} / \mathrm{m}^{3}\right)\end{array}$} & \multicolumn{2}{|c|}{ Unit fine aggregate content $\left(\mathrm{kN} / \mathrm{m}^{3}\right)$} & \multirow{2}{*}{$\begin{array}{c}\text { Unit coarse } \\
\text { aggregate } \\
\text { content }\left(\mathrm{kN} / \mathrm{m}^{3}\right)\end{array}$} & \multirow{2}{*}{$\begin{array}{c}\text { Admixture } \\
(\%)\end{array}$} \\
\hline & & & & & & Natural sand $\left(\mathrm{s}_{1}\right)$ & Crushed sand $\left(\mathrm{s}_{2}\right)$ & & \\
\hline 15.68 & 10 & 64.2 & 46.6 & 1.75 & 2.73 & 3.28 & 4.93 & 9.67 & 0.84 \\
\hline 15.68 & 15 & 64.2 & 47.6 & 1.86 & 2.90 & 3.27 & 4.92 & 9.26 & 0.89 \\
\hline 15.68 & 18 & 64.3 & 48.2 & 1.92 & 2.99 & 3.27 & 4.91 & 9.04 & 0.92 \\
\hline 17.64 & 10 & 59.3 & 45.7 & 1.74 & 2.94 & 3.19 & 4.79 & 9.75 & 0.9 \\
\hline 17.64 & 15 & 59.5 & 46.7 & 1.84 & 3.10 & 3.19 & 4.79 & 9.36 & 0.95 \\
\hline 17.64 & 18 & 59.5 & 47.3 & 1.91 & 3.21 & 3.19 & 4.77 & 9.10 & 0.98 \\
\hline 20.58 & 10 & 53.5 & 44.5 & 1.72 & 3.22 & 3.08 & 4.62 & 9.86 & 0.99 \\
\hline 20.58 & 15 & 53.4 & 45.5 & 1.83 & 3.43 & 3.07 & 4.60 & 9.44 & 1.05 \\
\hline 20.58 & 18 & 53.5 & 46.1 & 1.89 & 3.54 & 3.07 & 4.60 & 9.19 & 1.08 \\
\hline 23.52 & 10 & 48.6 & 43.5 & 1.72 & 3.53 & 2.97 & 4.46 & 9.91 & 1.08 \\
\hline 23.52 & 15 & 48.6 & 44.5 & 1.81 & 3.73 & 2.96 & 4.45 & 9.50 & 1.14 \\
\hline 23.52 & 18 & 48.6 & 45.1 & 1.87 & 3.85 & 2.96 & 4.44 & 9.25 & 1.18 \\
\hline 26.46 & 10 & 44.2 & 42.7 & 1.70 & 3.83 & 2.88 & 4.32 & 9.94 & 1.17 \\
\hline 26.46 & 15 & 44.3 & 43.7 & 1.80 & 4.07 & 2.87 & 4.30 & 9.49 & 1.25 \\
\hline 26.46 & 18 & 44.3 & 44.3 & 1.86 & 4.20 & 2.86 & 4.29 & 9.23 & 1.29 \\
\hline 29.40 & 10 & 40.9 & 42.0 & 1.69 & 4.13 & 2.80 & 4.19 & 9.93 & 1.26 \\
\hline 29.40 & 15 & 40.9 & 43.0 & 1.79 & 4.38 & 2.78 & 4.17 & 9.47 & 1.34 \\
\hline 29.40 & 18 & 40.9 & 43.6 & 1.85 & 4.53 & 2.77 & 4.16 & 9.21 & 1.39 \\
\hline 34.30 & 10 & 35.7 & 40.9 & 1.68 & 4.69 & 2.66 & 3.98 & 9.85 & 1.44 \\
\hline 34.30 & 15 & 35.7 & 41.9 & 1.77 & 4.97 & 2.64 & 3.96 & 9.39 & 1.52 \\
\hline 34.30 & 18 & 35.7 & 42.5 & 1.83 & 5.14 & 2.63 & 3.94 & 9.12 & 1.57 \\
\hline 39.20 & 10 & 32.1 & 40.2 & 1.67 & 5.19 & 2.55 & 3.82 & 9.72 & 1.59 \\
\hline 39.20 & 15 & 32.1 & 41.2 & 1.76 & 5.50 & 2.53 & 3.79 & 9.26 & 1.68 \\
\hline 39.20 & 18 & 32.1 & 41.8 & 1.82 & 5.67 & 2.51 & 3.77 & 8.99 & 1.74 \\
\hline
\end{tabular}

$$
\begin{gathered}
\text { net }_{p j}=\sum_{i} W_{j i} O_{p i}+\theta_{j} \\
O_{p j}=f_{j}\left(\text { net }_{p j}\right)
\end{gathered}
$$

where $f(\cdot)=$ activation function, which is generally taken as a sigmoid function:

$$
f(x)=1 /\left(1+e^{-x}\right)
$$

- Step 5: Calculate input values of an output layer $k$, net ${ }_{p k}$, using the output values of a hidden layer $j, O_{p i}$, connection weights $W_{k j}$, and biases $\theta_{k}$ between a hidden layer $j$ and an output layer $k$. Then, the output values of an output layer $k$,
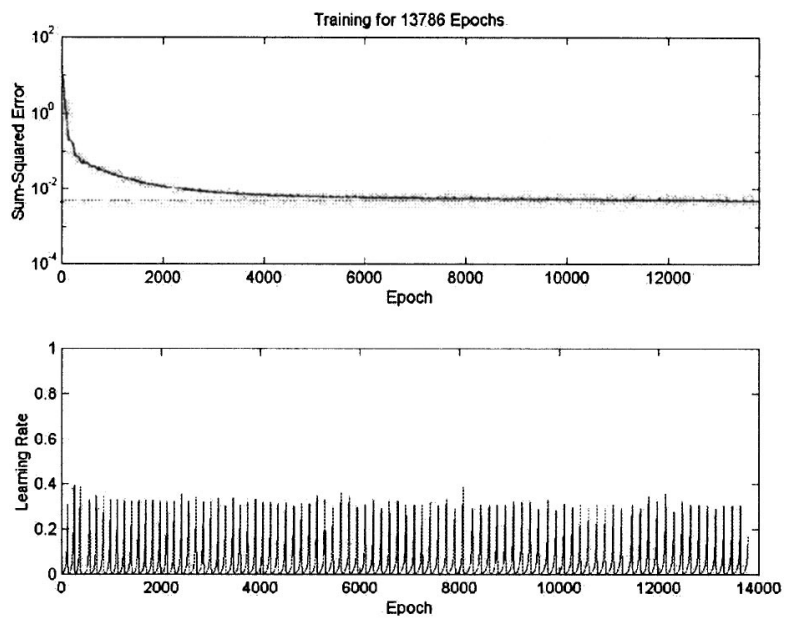

Fig. 2. Variation of sum-squared error and learning rate with training iterations in company A
$O_{p k}$, are derived from

$$
\begin{gathered}
\text { net }_{p k}=\sum_{j} W_{k j} O_{p j}+\theta_{k} \\
O_{p k}=f_{k}\left(\text { net }_{p k}\right)
\end{gathered}
$$

- Step 6: The error $E$ between the calculated value $O_{p k}$ and the desired value $T_{k}$ of an output layer may be defined as

$$
E=\frac{1}{2} \sum_{k=1}\left(O_{p k}-T_{k}\right)^{2}
$$

In the back-propagation networks, the error at the output neu-
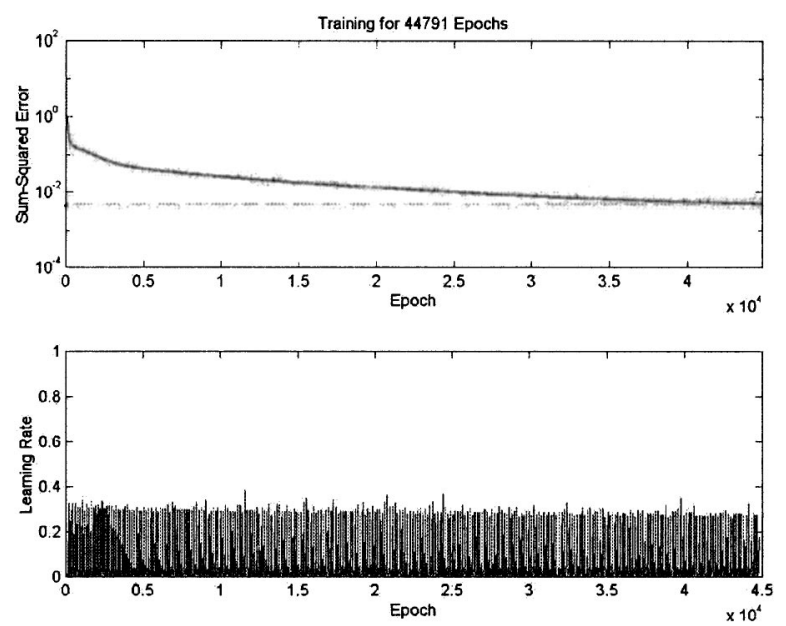

Fig. 3. Variation of sum-squared error and learning rate with training iterations in company B 
Table 3. Samples of Specified Concrete Mix Proportions of Company B for Training

\begin{tabular}{|c|c|c|c|c|c|c|c|c|c|}
\hline \multirow{2}{*}{$\begin{array}{l}\text { Specified } \\
\text { strength } \\
(\mathrm{MPa})\end{array}$} & \multirow{2}{*}{$\begin{array}{l}\text { Slump } \\
(\mathrm{cm})\end{array}$} & \multirow{2}{*}{$\begin{array}{l}\text { Water-cement } \\
\text { ratio }\end{array}$} & \multirow{2}{*}{$\begin{array}{c}\text { Fine } \\
\text { aggregate } \\
\text { percentage }(\%)\end{array}$} & \multirow{2}{*}{$\begin{array}{c}\text { Unit } \\
\text { water content } \\
\left(\mathrm{kN} / \mathrm{m}^{3}\right)\end{array}$} & \multirow{2}{*}{$\begin{array}{c}\text { Unit } \\
\text { cement content } \\
\left(\mathrm{kN} / \mathrm{m}^{3}\right)\end{array}$} & \multicolumn{2}{|c|}{ Unit fine aggregate content $\left(\mathrm{kN} / \mathrm{m}^{3}\right)$} & \multirow{2}{*}{$\begin{array}{c}\text { Unit coarse } \\
\text { aggregate } \\
\text { content }\left(\mathrm{kN} / \mathrm{m}^{3}\right)\end{array}$} & \multirow{2}{*}{$\begin{array}{l}\text { Admixture } \\
(\%)\end{array}$} \\
\hline & & & & & & Natural sand $\left(\mathrm{s}_{1}\right)$ & Crushed sand $\left(s_{2}\right)$ & & \\
\hline 15.68 & 10 & 63.1 & 50.9 & 1.68 & 2.66 & 9.23 & - & 9.08 & 1.36 \\
\hline 15.68 & 15 & 63.2 & 50.4 & 1.76 & 2.79 & 8.98 & - & 9.01 & 1.43 \\
\hline 15.68 & 18 & 63.1 & 50.1 & 1.81 & 2.87 & 8.83 & - & 8.96 & 1.47 \\
\hline 17.64 & 10 & 58.6 & 50.0 & 1.68 & 2.86 & 8.99 & - & 9.16 & 1.46 \\
\hline 17.64 & 15 & 58.5 & 49.5 & 1.75 & 3.00 & 8.74 & - & 9.14 & 1.53 \\
\hline 17.64 & 18 & 58.4 & 49.2 & 1.80 & 3.09 & 8.59 & - & 9.05 & 1.58 \\
\hline 20.58 & 10 & 52.6 & 48.8 & 1.66 & 3.15 & 8.68 & - & 9.29 & 1.61 \\
\hline 20.58 & 15 & 52.7 & 48.3 & 1.74 & 3.31 & 8.42 & - & 9.18 & 1.69 \\
\hline 20.58 & 18 & 52.6 & 48.0 & 1.78 & 3.39 & 8.29 & - & 9.15 & 1.73 \\
\hline 23.52 & 10 & 48.7 & 48.0 & 1.66 & 3.40 & 8.44 & - & 9.32 & 1.74 \\
\hline 23.52 & 15 & 48.8 & 47.5 & 1.73 & 3.56 & 8.19 & - & 9.23 & 1.82 \\
\hline 23.52 & 18 & 48.7 & 47.2 & 1.78 & 3.67 & 8.05 & - & 9.17 & 1.87 \\
\hline 26.46 & 10 & 45.2 & 47.3 & 1.65 & 3.65 & 8.23 & - & 9.35 & 1.86 \\
\hline 26.46 & 15 & 45.2 & 46.8 & 1.72 & 3.81 & 7.99 & - & 9.26 & 1.95 \\
\hline 26.46 & 18 & 45.3 & 46.5 & 1.77 & 3.92 & 7.84 & - & 9.19 & 2.00 \\
\hline 29.40 & 10 & 41.8 & 46.6 & 1.64 & 3.92 & 8.02 & - & 9.37 & 2.00 \\
\hline 29.40 & 15 & 41.7 & 46.1 & 1.72 & 4.12 & 7.76 & - & 9.25 & 2.10 \\
\hline 29.40 & 18 & 41.7 & 45.8 & 1.76 & 4.23 & 7.61 & - & 9.18 & 2.16 \\
\hline 34.30 & 10 & 37.0 & 45.7 & 1.63 & 4.40 & 7.69 & - & 9.32 & 2.25 \\
\hline 34.30 & 15 & 37.0 & 45.2 & 1.71 & 4.61 & 7.45 & - & 9.20 & 2.35 \\
\hline 34.30 & 18 & 37.0 & 44.9 & 1.75 & 4.74 & 7.29 & - & 9.11 & 2.42 \\
\hline 39.20 & 10 & 33.2 & 44.9 & 1.62 & 4.87 & 7.40 & - & 9.25 & 2.49 \\
\hline 39.20 & 15 & 33.2 & 44.4 & 1.71 & 5.14 & 7.11 & - & 9.08 & 2.62 \\
\hline 39.20 & 18 & 33.2 & 44.1 & 1.75 & 5.28 & 6.96 & - & 9.00 & 2.70 \\
\hline
\end{tabular}

rons is propagated backward to the hidden layer neurons, and then to the input layer neurons modifying the connection weights and the biases between them by a generalized delta rule. The modification of the weights and the biases in a generalized delta rule is used through a gradient descent of the error.

From the hidden to output neurons

$$
\Delta W_{k j}=\eta \delta_{k} O_{p j} \text { and } \Delta B_{k}=\eta \delta_{k}
$$

where $\delta_{k}=\left(T_{k}-O_{p k}\right) f^{\prime}\left(\right.$ net $\left._{p j}\right)$; and $\eta=$ learning rate.

And from input to hidden neurons

$$
\Delta W_{j i}=\eta \delta_{j} \text { net }_{p i} \text { and } \Delta B_{j}=\eta \delta_{j}
$$

where $\delta_{j}=W_{k j} \delta_{k} f^{\prime}\left(\right.$ net $\left._{p j}\right)$.

- Step 7: Repeat Steps 1 to 6 until error $E$ goes below a target value.

Table 4. Convergence in Training

\begin{tabular}{lccr}
\hline Experiment & Target error & Sum squared error & Epoch \\
\hline Company A & 0.10 & 0.0987907 & 143 \\
& 0.05 & 0.0499762 & 449 \\
& 0.01 & 0.0099989 & 4857 \\
& 0.005 & 0.0049999 & 13786 \\
Company B & 0.10 & 0.0999917 & 1765 \\
& 0.05 & 0.0499948 & 3159 \\
& 0.01 & 0.0099984 & 25184 \\
& 0.005 & 0.0049999 & 44791 \\
\hline
\end{tabular}

\section{Training of Neural Networks}

Concrete structures are generally required to have high safety, strength, durability, and serviceability. In order to produce highquality concrete to satisfy these needs, code information, specification, and experience of experts in determining concrete mix proportions play vital roles. The concretes used at construction sites are mostly produced by a ready-mixed concrete company according to specified concrete mix proportions. Generally, slump tests are performed before placing the concrete, but the compression tests of specimens are carried out at the 28th day after the placing. Therefore, it is difficult to estimate the compressive strength at construction sites. Ready-mixed concrete companies use their own mix proportions based on codes, previous experience, and experiments. In this study, the neural networks for es-

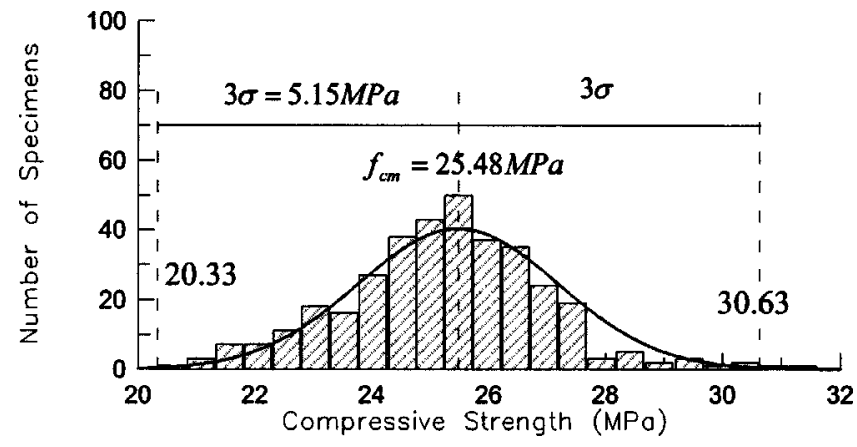

Fig. 4. Histogram of compressive strength of company A (180-12) 
Table 5. Data Sets of Specified Concrete Mix Proportions for Testing

\begin{tabular}{|c|c|c|c|c|c|c|c|c|c|c|}
\hline \multirow[b]{2}{*}{$\begin{array}{l}\text { Testing } \\
\text { data sets }\end{array}$} & \multirow{2}{*}{$\begin{array}{l}\text { Specified } \\
\text { Strength } \\
(\mathrm{MPa})\end{array}$} & \multirow[b]{2}{*}{$\begin{array}{c}\text { Slump } \\
(\mathrm{cm})\end{array}$} & \multirow[b]{2}{*}{$\begin{array}{c}\text { Water-cement } \\
\text { ratio }\end{array}$} & \multirow{2}{*}{$\begin{array}{c}\text { Fine } \\
\text { aggregate } \\
\text { percentage }(\%)\end{array}$} & \multirow{2}{*}{$\begin{array}{c}\text { Unit } \\
\text { water } \\
\text { content } \\
\left(\mathrm{kN} / \mathrm{m}^{3}\right) \\
\end{array}$} & \multirow{2}{*}{$\begin{array}{c}\text { Unit } \\
\text { cement } \\
\text { content } \\
\left(\mathrm{kN} / \mathrm{m}^{3}\right)\end{array}$} & \multicolumn{2}{|c|}{ Unit fine aggregate content $\left(\mathrm{kN} / \mathrm{m}^{3}\right)$} & \multirow{2}{*}{$\begin{array}{c}\text { Unit } \\
\text { coarse aggregate } \\
\text { content }\left(\mathrm{kN} / \mathrm{m}^{3}\right)\end{array}$} & \multirow[b]{2}{*}{$\begin{array}{c}\text { Admixture } \\
(\%) \\
\end{array}$} \\
\hline & & & & & & & $\begin{array}{c}\text { Natural } \\
\text { sand }\left(s_{1}\right)\end{array}$ & $\begin{array}{c}\text { Crushed } \\
\text { sand }\left(\mathrm{s}_{2}\right)\end{array}$ & & \\
\hline \multirow[t]{10}{*}{ Company A } & 17.64 & 8 & 59.4 & 45.3 & 1.71 & 2.87 & 3.19 & 4.79 & 9.91 & 0.88 \\
\hline & 20.58 & 8 & 53.6 & 44.1 & 1.69 & 3.15 & 3.08 & 4.63 & 10.03 & 0.96 \\
\hline & 23.52 & 8 & 48.6 & 43.1 & 1.67 & 3.43 & 2.98 & 4.47 & 10.10 & 1.05 \\
\hline & 26.46 & 8 & 44.4 & 42.3 & 1.66 & 3.73 & 2.89 & 4.33 & 10.11 & 1.10 \\
\hline & 29.40 & 8 & 40.9 & 41.6 & 1.65 & 4.03 & 2.80 & 4.20 & 10.10 & 1.31 \\
\hline & 17.64 & 12 & 59.5 & 46.1 & 1.78 & 3.00 & 3.19 & 4.79 & 9.59 & 0.92 \\
\hline & 20.58 & 12 & 53.6 & 44.9 & 1.76 & 3.29 & 3.08 & 4.62 & 9.70 & 1.01 \\
\hline & 23.52 & 12 & 48.6 & 43.9 & 1.75 & 3.61 & 2.97 & 4.45 & 9.74 & 1.10 \\
\hline & 26.46 & 12 & 44.3 & 43.1 & 1.74 & 3.94 & 2.87 & 4.31 & 9.74 & 1.21 \\
\hline & 29.40 & 12 & 40.9 & 42.4 & 1.72 & 4.21 & 2.79 & 4.19 & 9.75 & 1.29 \\
\hline \multirow[t]{10}{*}{ Company B } & 17.64 & 8 & 58.6 & 50.2 & 1.64 & 2.79 & 9.10 & - & 9.20 & 1.43 \\
\hline & 20.58 & 8 & 52.5 & 49.0 & 1.63 & 3.10 & 8.78 & - & 9.31 & 1.58 \\
\hline & 23.52 & 8 & 48.7 & 48.2 & 1.62 & 3.32 & 8.56 & - & 9.37 & 1.70 \\
\hline & 26.46 & 8 & 45.2 & 47.5 & 1.62 & 3.58 & 8.33 & - & 9.39 & 1.83 \\
\hline & 29.40 & 8 & 41.7 & 46.8 & 1.61 & 3.85 & 8.11 & - & 9.47 & 1.97 \\
\hline & 17.64 & 12 & 58.6 & 49.8 & 1.71 & 2.91 & 8.90 & - & 9.14 & 1.49 \\
\hline & 20.58 & 12 & 52.6 & 48.6 & 1.70 & 3.22 & 8.57 & - & 9.24 & 1.65 \\
\hline & 23.52 & 12 & 48.7 & 47.8 & 1.69 & 3.46 & 8.35 & - & 9.29 & 1.77 \\
\hline & 26.46 & 12 & 45.2 & 47.1 & 1.68 & 3.70 & 8.14 & - & 9.32 & 1.89 \\
\hline & 29.40 & 12 & 41.7 & 46.4 & 1.68 & 4.00 & 7.92 & - & 9.33 & 2.04 \\
\hline
\end{tabular}

timating the concrete compressive strength were trained and tested by actual mix proportion data provided by two companies, $\mathrm{A}$ and B. The material properties of concrete from the two companies are shown in Table 1. Normal Portland cement was used. The maximum size of the aggregate is $25 \mathrm{~mm}$, the range of compressive strengths is from 9.8 to $39.2 \mathrm{MPa}$, and slump values are $5,8,12,15,18$, and $21 \mathrm{~cm}$.

Nine (for company B) or ten (for company A) parameters, including the specified compressive strength, water-cement ratio, fine aggregate percentage, unit water content, unit cement content, unit fine aggregate content, unit coarse aggregate content, admixtures and slump, were used for the training and learning of the neural networks, where the first parameter is the output and the rest the input. Company A used a mixture of natural sand $\left(\mathrm{s}_{1}\right)$ and crushed sand $\left(\mathrm{s}_{2}\right)$ while Company $\mathrm{B}$ used only the natural sand, so company A has an additional unit fine aggregate content parameter. In total, 98 data sets were used to train the neural networks for each of the two companies. Tables 2 and 3 show the samples of these data sets.

During the training of the neural networks, the connection weights and biases of the networks were updated following the seven-step procedures described in the subsection entitled "Overview of Neural Networks," until the sum of the squared error $E$ was less than a required target error. In order to investigate the computational convergence, the estimation of the compressive strength was carried out for different target errors: $0.10,0.05$, 0.01 , and 0.005 . Figs. 2 and 3 display training convergences of companies $\mathrm{A}$ and $\mathrm{B}$ as examples under the target error of 0.005 . The neural networks applied to estimate the compressive strength of concrete converged very well. The rate of convergence was very fast in the early stage, but gradually became slower as the epoch continued. Table 4 shows the relation of convergence in

Table 6. Estimated Values and Error Percentages in Company A

\begin{tabular}{|c|c|c|c|c|c|c|}
\hline \multirow[b]{2}{*}{ Testing data set } & \multirow{2}{*}{$\begin{array}{l}\text { Slump } \\
(\mathrm{cm})\end{array}$} & \multirow{2}{*}{$\begin{array}{c}\text { Specified } \\
\text { strength (MPa) }\end{array}$} & \multicolumn{4}{|c|}{ Predicted compressive strengths (MPa) } \\
\hline & & & Target error 0.10 & Target error 0.05 & Target error 0.01 & Target error 0.005 \\
\hline 1 & 8 & 17.64 & $18.13(2.8)$ & $17.54(0.6)$ & $17.54(0.6)$ & $17.84(1.1)$ \\
\hline 2 & 8 & 20.58 & $21.76(5.7)$ & $20.19(1.9)$ & $20.19(1.9)$ & $20.58(0.0)$ \\
\hline 3 & 8 & 23.52 & $24.40(3.8)$ & $23.23(1.3)$ & $23.42(0.4)$ & $23.42(0.4)$ \\
\hline 4 & 8 & 26.46 & $26.07(1.5)$ & $25.58(3.3)$ & $26.17(1.1)$ & $25.77(2.6)$ \\
\hline 5 & 8 & 29.40 & $29.50(0.3)$ & $30.87(5.0)$ & $30.18(2.7)$ & $29.99(2.0)$ \\
\hline 6 & 12 & 17.64 & $16.86(4.4)$ & $17.64(0.0)$ & $17.64(0.0)$ & $17.74(0.6)$ \\
\hline 7 & 12 & 20.58 & $20.29(1.4)$ & $20.48(0.5)$ & $20.48(0.5)$ & $20.68(0.5)$ \\
\hline 8 & 12 & 23.52 & $23.91(1.7)$ & $23.62(0.4)$ & $23.32(0.8)$ & $23.72(0.8)$ \\
\hline 9 & 12 & 26.46 & $27.15(2.6)$ & $27.34(3.3)$ & $26.36(0.4)$ & $26.36(0.4)$ \\
\hline 10 & 12 & 29.40 & $29.60(0.7)$ & $30.18(2.7)$ & $29.60(0.7)$ & $28.71(2.3)$ \\
\hline
\end{tabular}

Note: The numerical values in parentheses present the percent differences between the specified and the predicted strengths. 
Table 7. Estimated Values and Error Percentages in Company B

\begin{tabular}{|c|c|c|c|c|c|c|}
\hline \multirow[b]{2}{*}{ Testing data set } & \multirow[b]{2}{*}{ Slump $(\mathrm{cm})$} & \multirow[b]{2}{*}{ Specified strength (MPa) } & \multicolumn{4}{|c|}{ Predicted compressive strengths $(\mathrm{MPa})$} \\
\hline & & & Target error 0.10 & Target error 0.05 & Target error 0.01 & Target error 0.005 \\
\hline 1 & 8 & 17.64 & $17.05(3.3)$ & $16.76(5.0)$ & $17.35(1.7)$ & $17.44(1.1)$ \\
\hline 2 & 8 & 20.58 & $20.58(0.0)$ & $20.68(0.5)$ & $20.68(0.5)$ & $20.78(0.9)$ \\
\hline 3 & 8 & 23.52 & $24.01(2.1)$ & $24.01(2.1)$ & $23.72(0.8)$ & $23.72(0.8)$ \\
\hline 4 & 8 & 26.46 & $26.75(1.1)$ & $26.66(0.7)$ & $26.46(0.0)$ & $26.36(0.4)$ \\
\hline 5 & 8 & 29.40 & $30.38(3.3)$ & $30.67(4.3)$ & $29.99(2.0)$ & $29.40(0.0)$ \\
\hline 6 & 12 & 17.64 & $16.66(5.6)$ & $16.56(6.1)$ & $17.25(2.2)$ & $17.35(1.7)$ \\
\hline 7 & 12 & 20.58 & $20.09(2.4)$ & 20.29 (1.4) & $20.48(0.5)$ & $20.68(0.5)$ \\
\hline 8 & 12 & 23.52 & $23.42(0.4)$ & $23.42(0.4)$ & $23.42(0.4)$ & $23.52(0.0)$ \\
\hline 9 & 12 & 26.46 & $26.85(1.5)$ & $26.66(0.7)$ & $26.56(0.4)$ & $26.56(0.4)$ \\
\hline 10 & 12 & 29.40 & $29.69(1.0)$ & $29.40(0.0)$ & $29.50(0.3)$ & $29.30(0.3)$ \\
\hline
\end{tabular}

Note: The numerical values in parentheses present the percent differences between the specified and the predicted strengths.

training by neural networks. For company A, with the target errors decreasing from 0.05 to 0.005 , the epochs increased 3.1, 34.0, and 96.4 times than that of a target error, 0.10. For company $\mathrm{B}$, the epochs increased $1.8,14.3$, and 25.4 times than that of a target error, 0.10. This means that the amount of calculations increase with geometric series to obtain more precise results.

Once trained, the neural networks were tested using ten data sets shown in shown in Table 5. Again, these data sets are actual data provided by Companies $\mathrm{A}$ and $\mathrm{B}$. In the testing process of the neural networks, the specified compressive strength of concrete were estimated using the same nine input parameters as those in training.

\section{Testing of Neural Networks}

Once trained, the neural networks need to be tested to evaluate whether they can successfully estimate the compressive strength of concrete based on mixing proportions. The ten data sets shown in Table 5 were used for testing. Again they are actual data provided by Companies A and B, but completely different from those used for training the neural networks. In the testing process, the data representing the water-cement ratio, fine aggregate percentage, unit water content, unit cement content, unit fine aggregate content $\left(\mathrm{s}_{1}, \mathrm{~s}_{2}\right)$, unit coarse aggregate, admixture, and slump were inputted to the neural networks, and the compressive strength of the concrete were estimated as output of the neural network.

The compressive strengths of concrete estimated by the trained neural networks are listed in Tables 6 and 7. For the company A results listed in Table 6 , the range of error percentages of the estimated strength compared to the specified strength by mix proportions show $0.3-5.7,0.0-5.0,0.0-2.7$, and $0.0-2.7$ percents, respectively, for target errors of $0.1,0.05,0.01$, and 0.005 . For the company B results listed in Table 7 , the range of error percentages shows $0.0-5.6,0.0-6.1,0.0-2.2$, and $0.0-1.7$ percents, respectively, for target errors of $0.1,0.05,0.01$, and 0.005 .

The errors of the estimated strength compared to the required strength do not decrease beyond a certain level of target error while computation time dramatically increased. Therefore, it is important to determine an optimal target error for computational efficiency. In this study, the target error was determined to be 0.01 .

\section{Comparison of Estimated and Tested Strengths}

In order to verify the effectiveness of the neural networks in estimating the compressive strengths of concrete based on the mix proportion parameters, the strengths estimated by the neural networks were compared with the results from the actual compression tests carried out by the two companies. The results of the compression tests may be affected by the type of test specimens, specimen size, the type of molds, curing conditions, the preparation of end surfaces, the rigidity of a testing machine, and the rate of application of stress. Aside from the variations in the type of Portland cement, admixtures, source of aggregates, mix proportions, batching, mixing and delivery also have an influence on the compressive strength of concrete. In these tests, the specified strengths of concrete were 17.64, 20.58, and $23.52 \mathrm{MPa}$ and slump was $12 \mathrm{~cm}$. Cylindrical specimens with dimensions of $\phi$ $100 \times 200 \mathrm{~mm}$ were tested, which were made according to the mix proportions shown in Tables 2 and 3. The tests followed the requirements of KS F2405 (1997) and ASTM C39-93a at an age of 28 days. The results of the tests are shown in Table 8 , in which the coefficient of increase $(\alpha)$ in mix design is predetermined as

Table 8. Results of Compression Tests

\begin{tabular}{|c|c|c|c|c|c|c|c|c|c|}
\hline \multirow[b]{2}{*}{ Experiment } & \multirow{2}{*}{$\begin{array}{c}\text { Specified } \\
\text { strength } \\
\left(f_{c}^{\prime}, \mathrm{MPa}\right) \\
\end{array}$} & \multirow{2}{*}{$\begin{array}{l}\text { Slump } \\
(\mathrm{cm})\end{array}$} & \multirow{2}{*}{$\begin{array}{l}\text { Number } \\
\text { of tests }\end{array}$} & \multirow{2}{*}{$\begin{array}{l}\text { Standard } \\
\text { deviation } \\
(\sigma, \mathrm{MPa})\end{array}$} & \multirow[b]{2}{*}{$t$} & \multicolumn{2}{|c|}{ Coefficient of increase $(\alpha)$} & \multirow{2}{*}{$\begin{array}{c}\text { Average } \\
\text { strength } \\
\left(f_{c m}=f_{c r}, \mathrm{MPa}\right)\end{array}$} & \multirow{2}{*}{$\begin{array}{c}\text { Specified } \\
\text { strength by tests } \\
\left(f_{c 1}^{\prime}, \mathrm{MPa}\right) \\
\end{array}$} \\
\hline & & & & & & Design & Test & & \\
\hline \multirow[t]{3}{*}{ Company A } & 17.64 & 12 & 447 & 1.26 & 3.0 & 1.22 & 1.26 & 22.15 & $18.33(3.9)$ \\
\hline & 20.58 & 12 & 354 & 1.72 & 3.0 & 1.22 & 1.24 & 25.48 & $20.38(1.0)$ \\
\hline & 23.52 & 12 & 372 & 1.77 & 3.0 & 1.22 & 1.24 & 29.20 & $23.91(1.7)$ \\
\hline \multirow[t]{3}{*}{ Company B } & 17.64 & 12 & 345 & 1.11 & 3.0 & 1.20 & 1.19 & 20.97 & $17.64(0.0)$ \\
\hline & 20.58 & 12 & 435 & 1.59 & 3.0 & 1.20 & 1.23 & 25.38 & $20.58(0.0)$ \\
\hline & 23.52 & 12 & 363 & 1.62 & 3.0 & 1.20 & 1.22 & 28.71 & $23.91(1.7)$ \\
\hline
\end{tabular}

Note: The numerical values in parentheses present the percent differences between the specified strength by test results and the specified strength on mix proportion. 


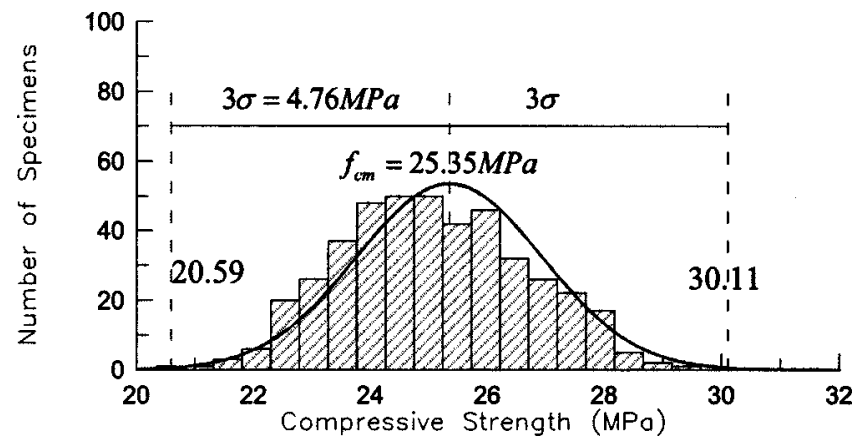

Fig. 5. Histogram of compressive strength of company B (180-12)

the ratio of required average strength $\left(f_{c r}\right)$ to specified strength $\left(f_{c 1}^{\prime}\right)$ for various coefficients of variation and the chances of falling below specified strength (ACI 214 1989). The coefficient of increase $(\alpha)$ in the tests is calculated as follows:

$$
\alpha=f_{c m} / f_{c 1}^{\prime}
$$

where $f_{c m}=$ average strength of test results and equals to $f_{c r}$; and $f_{c 1}^{\prime}=$ specified strength by test results. The specified strength $\left(f_{c 1}^{\prime}\right)$ from the test results was derived as follows:

$$
f_{c 1}^{\prime} \leqslant f_{c r}-t \sigma
$$

where $\sigma=$ forecast value of the standard deviation; $f_{c r}=$ required average strength; and $t=$ coefficient to be determined by the probability that may fall below $f_{c}^{\prime}$ according to ACI 214. In this study, $t$ was taken as 3.0 since none of the test results fell below the limit $f_{c}^{\prime}$. Comparing the specified strengths in Table 8, the maximum percentage of the differences between the specified strength by tests $\left(f_{c 1}^{\prime}\right)$ and the specified strength in concrete mix proportions $\left(f_{c}^{\prime}\right)$ is $3.9 \%$. This means that the results of the tests show good agreement with the specified strengths.

Figs. 4 and 5 show the histograms of the test results for a specified compressive strength $20.58 \mathrm{MPa}$ and slump $12 \mathrm{~cm}$. The numbers of test specimens are 354 and 453, respectively, for companies A and B. In Figs. 4 and 5, the test results show normal distributions and the standard deviations are $1.715 \mathrm{MPa}$ for company $\mathrm{A}$ and $1.588 \mathrm{MPa}$ for company $\mathrm{B}$. The strengths obtained from the tests are 20.38 MPa for company A and 20.58 MPa for company B. The strengths from the tests show good agreement with those of the concrete mix proportions. Since the histograms of another set of testing results show trends similar to Figs. 4 and 5 , they are not presented in this paper.
Table 8 shows the comparison of the strengths estimated by the neural networks (at the target error of 0.01) to the test results. In Table 9, the required average strength in the mix proportions is computed by multiplying the coefficient of increase $\alpha$ (as given in Table 8) to the specified strength. In the specified strengths, the range of error percent differences between the test and the estimated results compared to the estimated values are $0.5 \%-3.9 \%$ for company A and $0.5 \%-2.3 \%$ for company B. In the required average strengths, the range of error percent differences between the test and the estimated results compared to the estimated values are $1.7-2.7 \%$ for company A and $1.4-3.2 \%$ for company B. Therefore, the compressive strengths of concrete estimated by the proposed neural networks agree with those resulting from compressive tests, that demonstrates the effectiveness of the neural networks.

\section{Conclusions}

This paper presented the application of neural networks to estimate the compressive strength of concrete based on its mix proportion parameters, such as water-cement ratio, fine aggregate percentage, unit water content, unit cement content, unit fine aggregate content, unit coarse aggregate, admixture, and slump. The neural networks were developed and trained using the concrete mix proportion data from two ready-mixed concrete companies and the compressive strength of the concrete was estimated by the trained neural networks. In this study, the optimal target error for training the neural networks was found to be 0.01 for both computational accuracy and efficiency. The validity of the proposed neural network-based technique was proven by comparing the estimated strength with the compressive testing results of the concrete specimen provided by the two companies. The maximum errors between the estimated and tested results were $3.9 \%$ in the specified strengths and $3.2 \%$ in the required average strengths.

This study demonstrated the effectiveness of the neural network-based technique in estimating the compressive strength of concrete based on concrete mix proportion parameters before the placement of concrete. As a future study, other important data that also affect the concrete strength, such as the uncertainty of concrete (i.e., the quality variation of aggregate and cement, measuring error, mixing condition, etc.) and in-field conditions (i.e., delivery distance, curing condition, etc.) will be collected and considered in the neural networks. As more data are accumulated over the time, the neural networks trained by these data will become more effective and the resulting estimation will become more accurate and reliable.

\begin{tabular}{|c|c|c|c|c|c|c|}
\hline \multirow[b]{2}{*}{ Experiment } & \multicolumn{3}{|c|}{ Specified strengths (MPa) } & \multicolumn{3}{|c|}{ Required average strengths (MPa) } \\
\hline & Design & Test & Estimation & Design & Test & Estimation \\
\hline \multirow[t]{3}{*}{ Company A } & 17.64 & 18.33 & $17.64(3.9)$ & 21.56 & 22.15 & $21.56(2.7)$ \\
\hline & 20.58 & 20.38 & $20.48(0.5)$ & 25.09 & 25.48 & $24.99(2.0)$ \\
\hline & 23.52 & 23.91 & $23.32(2.5)$ & 28.71 & 29.20 & $28.71(1.7)$ \\
\hline \multirow[t]{3}{*}{ Company B } & 17.64 & 17.64 & $17.25(2.3)$ & 21.17 & 20.97 & $20.68(1.4)$ \\
\hline & 20.58 & 20.58 & $20.48(0.5)$ & 24.70 & 25.38 & $24.60(3.2)$ \\
\hline & 23.52 & 23.91 & $23.42(2.1)$ & 28.22 & 28.71 & $28.13(2.1)$ \\
\hline
\end{tabular}

Table 9. Comparison of the Specified and the Required Average Strengths

Note: The numerical values in parentheses present the percent differences between the test and the estimated values. 


\section{Acknowledgments}

This research was supported by Taegu University and University of California, Irvine. The writers cordially express their gratitude for the support.

\section{Notation}

The following symbols were used in this paper:

$E=$ error between $O_{p k}$ and $T_{k}$;

$f(\cdot)=$ sigmoid function;

net $_{p i}=$ input value of input neurons;

net $_{p j}=$ input value of hidden neurons;

net $_{p k}=$ input value of output neurons;

$O_{p j}=$ activation value of hidden neurons;

$O_{p k}=$ activation value of output neurons;

$T_{k}=$ desired value of output neurons;

$W_{j i}, W_{k j}=$ connection strengths; and

$\theta_{j}, \theta_{k}=$ biases.

\section{References}

Adeli, H., and Park, H. S. (1995). "A neural dynamic model for structural optimization-Theory." Comput. Struct., 57(3), 383-390.

American Concrete Institute (ACI). (1989). "Recommended practice for evaluation of strength test results of concrete." Rep. No. ACI214-89, Detroit.

Chen, H. M., Tsai, K. H., Qi, G. Z., Yang, C. S., and Amini, F. (1995) "Neural network for structure control." J. Comput. Civ. Eng., 9(2), $168-176$

Chen, S. S., and Shah, K. (1992). "Neural networks in dynamic analysis of bridges." Proc., 8th Conf. Computing in Civil Engineering, ASCE, New York, 1058-1065.

Feng, M. Q., and Bahng, E. Y. (1999). "Damage assessment of jacketed RC columns using vibration tests." J. Struct. Eng., 125(3), 265-271.

Feng, M. Q., and Kim, J. M. (1998). "Identification of a dynamic system using ambient vibration measurements." J. Appl. Mech., 65(2), 10101023.

Oh, J. W., Lee, I. W., Kim, J. T., and Lee, G. W. (1999). “Application of neural networks for proportioning of concrete mixes." ACI Mater. J., 96(1), 61-67. 